\title{
CPLD-PGPS Scheduler in Wireless OFDM Systems
}

\author{
Zhifeng Diao, Student Member, IEEE, Dongxu Shen, Member, IEEE, and Victor O. K. Li, Fellow, IEEE
}

\begin{abstract}
In this paper, we propose a new scheduler for orthogonal frequency-division multiplexing (OFDM) wireless communication systems, called Channel-Condition and Packet-Length Dependent Packet Generalized Processor Sharing (CPLD-PGPS) scheduler. Based on PGPS, the CPLD scheduler considers both the physical channel condition and the length of packets, and optimally allocates the sub-carriers to different users. The total transmit power is adaptively allocated to each subcarrier. With this scheduler, the system can achieve better system BER performance, and correspondingly superior PER performance. The system throughput is improved, the required bandwidth is guaranteed, and long term fairness for all traffic in the system is provided. In order to reduce the complexity, a simplified algorithm is proposed, which maintains the system throughput as in the original scheduler, and guarantees the system performance with properly set system parameters. The superior performance of the proposed schedulers is demonstrated by simulation with multimedia traffic.
\end{abstract}

Index Terms - Channel condition, fairness, OFDM, packet length, PGPS, throughput.

\section{INTRODUCTION}

$I^{N}$ $\mathrm{N}$ wireless networks, packet scheduling plays a key role in resource allocation. Many schemes for packet scheduling in wireless networks have emerged, based on the concept of fair queuing. The original idea of fair scheduling, generalized processor sharing (GPS) is proposed in [1] for wire-line networks. The basic idea of GPS is to assign each user a fixed weight, instead of a fixed bandwidth, and dynamically allocate bandwidth to all users according to the assigned weight and traffic load. Packet generalized processor sharing (PGPS) is proposed in [2]. The GPS-based fair schedulers proposed for wire-line packet networks have been adapted to wireless networks. Unlike wire-line networks, the packets transmitted on wireless channels are subject to burst errors, and the bandwidth is more scarce.

For wireless networks, an ideal fair-scheduling algorithm is provided in [3], which is a packetized implementation of the fluid model by considering wireless channel information. In [4], Cao et al. give a novel wireless scheduling algorithm for delay-sensitive and best-effort traffic, which can guarantee the QoS requirement of each traffic class with fairness considerations. In [5], a channel state dependent packet scheduler is proposed to enhance the system performance, but no bandwidth and fairness guarantees are provided. In [6], wireless

Manuscript received October 18, 2004; revised July 28, 2004 and October 15, 2004; accepted November 27, 2005. The associate editor coordinating the review of this paper and approving it for publication was L.-C. Wang.

Z. Diao is with the Department of Electrical Engineering, The University of California, Riverside, 221 Engineering Bldg Unit 2, Riverside, CA 92521 USA (email: zdiao@ee.ucr.edu).

D. Shen and V. Li are with the Department of Electrical and Electronic Engineering, The University of Hong Kong, Hong Kong, China (email: dxshen@eee.hku.hk; vli@eee.hku.hk).

Digital Object Identifier 10.1109/TWC.2006.04685 scheduling algorithms such as channel state dependent packet scheduler (CSDPS), server-based fair approach (SBFA), etc., are examined. Other work on the wireless scheduling algorithm design includes [7] and [8].

Some work has integrated wireless scheduling algorithms into time-division multiple access (TDMA) and code-division multiple access (CDMA) systems. For wireless TDMA systems, [9] introduces scheduling policies to optimize the overall system performance, and to satisfy the maximum tolerable packet delay and dropping probability requirements. In [10], Zhuge et al. propose a packet scheduling algorithm in cellular CDMA systems, which is adaptive not only to packet delay deadline required by the application, but also the deviation of data rate from its target value and the channel status variations. [11], [12] provide dynamic packet scheduling algorithms in cellular CDMA systems with QoS constraints, and the system performance is improved, while the fairness is also provided.

OFDM is considered to be the next generation wireless telecommunication system, which divides the frequency channel into a number of subcarriers [13]. The scheduling algorithm plays an important role in wireless OFDM systems. OFDM systems are different from TDMA systems that allocate resource only in the time domain. So these scheduling algorithms proposed for TDMA can not be applied to OFDM systems directly. For multi-user OFDM systems, the way subcarriers are allocated to all users also affects the overall system performance. [14] gives an optimal algorithm to adaptively allocate the subchannels to the users. Then a heuristic algorithm based on constructive assignment and iterative improvement is proposed, which is valid for the real system. To minimize the total transmit power, Cheng et al. [15] propose a new algorithm to allocate the subcarriers and transmit power among all users in frequency selective fading environments, and adaptive modulation on each subcarrier is also considered. [16] divides all subcarriers into subbands. According to the principle of Multiple Carrier Proportional Fairness (MCPF), all subbands are allocated to the users. [17] also considers dynamic resource management in OFDM systems. Nearly all these resource allocation schemes only consider bit level scheduling. In order to guarantee fairness and achieve a good system throughput, a metric on the efficiency of using transmission power [22] is introduced and the proportional-fair power allocation scheme is proposed.

Many researchers have studied packet scheduling in OFDM systems. [18] introduces a resource management scheme for downlink packet transmission. Based on GPS scheduler, a truncated GPS (TGPS) scheduler is proposed to maximize the system throughput and achieve the QoS requirements of users. However, the packet length is fixed, which is unrealistic in practical systems. For wideband wireless systems, [19] 
summarizes the dynamic packet assignment for the media access control layer, but the packet assignment scheme does not consider the physical characteristics of OFDM systems.

We consider a system in which a number of OFDM symbols are grouped into an OFDM data frame, and packets with variable length are transferred in the frame. Since the number of good sub-channels of each user is variable, when a packet is to be accommodated in the current OFDM frame, the number of good subchannels is much less than the number of subchannels this packet needs. Even though the subcarrier allocation is optimized, this packet may still have a high probability to experience errors. The system performance strongly depends on the channel condition and the packet length.

In this paper, we propose a new packet scheduler based on user channel condition and packet length (CPLD-PGPS). The total transmission power is constrained, and is adaptively allocated among the users and the subcarriers. By considering the subchannel conditions and the length of the packet together, the CPLD scheduler is able to guarantee the required bandwidth, the system packet error rate performance, and the system throughput. Then a simplified CPLD scheduler is given, which can guarantee the system performance by properly setting parameters. The physical channels around the base station are assumed to have nearly the same statistical property. This scheduler can also provide fairness in the long term. In the simulation, the packet length is set to be an integral multiple of the number of bits a subcarrier in a frame can accommodate. Simulation results show that the proposed scheduler can achieve better PER performance, system throughput, and packet delay than the conventional PGPS scheduler.

The remainder of this paper is organized as follows. In Section II, the OFDM system architecture is introduced. The algorithm of CPLD scheduler is given in Section III. Then the scheduler is simplified in Section IV. The simulation results are shown in Section V. The conclusions are made in Section VI.

\section{OFDM System ARChitecture}

In this section, we give the wireless channel model and describe the OFDM system architecture. To support multimedia transmission over wireless channels, this system allows users transmitting speech, video and data. We denote the transmission of a particular media as a session and each user only has one session at a time. Packet scheduling is performed centrally at the base station (BS), and we focus on the downlink performance. Medium Access Control is integrated with packet scheduling. The scheduler is assumed to have perfect knowledge of the channel. The total transmission power is constrained, and is optimally allocated to each user.

\section{A. Channel Model}

We assume the multipath fading channel is wide sense stationary with uncorrelated scattering. With tolerable leakage, the time domain channel impulse response is modeled as a tapped delay line at a tap spacing of one sampling interval. In the time domain, the channel impulse response can be expressed as

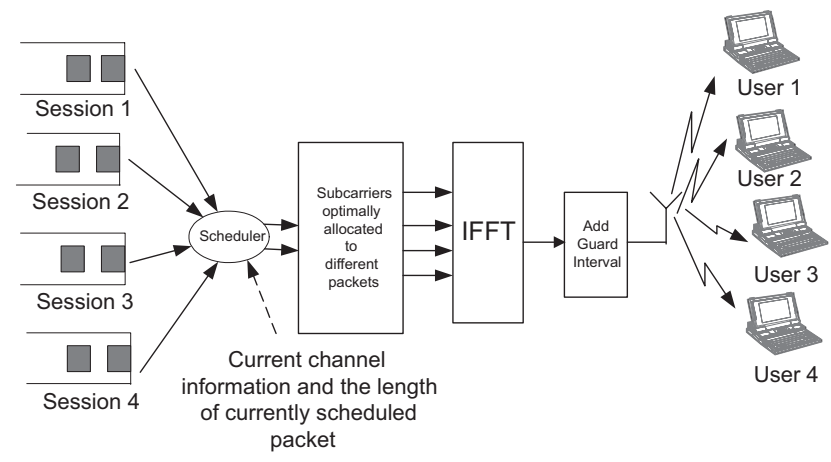

Fig. 1. The OFDM system description.

$$
h(t, \tau)=\sum_{i=1}^{S} \alpha_{i}(t) \delta\left(t-\tau_{i}\right)
$$

where $\alpha_{i}(t)$ is the complex gain of path $i, \tau_{i}$ is the corresponding path delay, $S$ is the number of paths, and $\delta(\tau)$ is the Dirac delta function. Here $\tau_{i}=i \Delta t$, where $\Delta t$ is the sampling interval of the OFDM system.

In the frequency domain, the channel response is represented as

$$
H(n, j)=\sum_{i=1}^{S} a_{i}\left(n T_{s}\right) W^{i j}
$$

where $n$ is the index for an OFDM symbol, $j$ is the subcarrier index, $T_{s}$ is the duration of an OFDM symbol, $W=e^{-j 2 \pi / N_{c}}$, and $N_{c}$ is the number of OFDM subcarriers.

\section{B. System Description}

The system description is given in Fig. 1. This system consists of one cell with one base station (BS) communicating simultaneously with $K$ mobile terminals. All the mobile terminals are randomly distributed in the cell. We group $N_{s}$ OFDM symbols as a frame. Each subcarrier in the frame can only be allocated to one packet. All the subcarriers have the same modulation scheme. The base station establishes an individual queue for each user. Arriving packets from each user is stored in its own individual queue in a FIFO (first-in first-out) manner. The system monitors the downlink channel condition for each mobile user. Based on the virtual finishing time of PGPS, and according to the current channel condition and the length of the packet, the packet scheduler decides which user's packet should be transmitted. The system will allocate the subcarriers to different selected packets based on the channel conditions of all users. The whole subcarrier can only be allocated to one packet, and according to channel conditions, the subcarriers are optimally allocated to achieve a better PER performance. After inverse fast fourier transform (IFFT) and the addition of a guard interval, the frame is sent out. The subcarrier allocation map is transferred to the users by another control channel. According to the subcarrier allocation map, a user will judge whether there are packets in the received frame: if yes, the packet is extracted from the frame. 
We assume the channels between users and the BS are independent with the same statistical property. Without loss of generality, we assume the average channel gain is 1 , and all the receivers have the same noise figure. If the energy $E_{s}$ of the subchannel is larger than $E_{0}$, (the value $E_{0}$ is set by the system), the subcarrier is regarded as a good subchannel; otherwise, it is a bad subchannel. We assume that BS can obtain perfect knowledge of the channel information of all users from the pilot subcarriers and the total transmission power is fixed.

\section{CPLD-PGPS SCHEDULING IN OFDM SYSTEMS}

\section{A. Virtual Time Update}

Generalized processor sharing (GPS) assigns each data flow a weight so that the amount of processor power received by each flow is proportional to its weight. GPS is an ideal scheduling policy that assumes packets are infinitely divisible. To apply GPS to a server with different arriving streams, each stream is allocated an infinite buffer and only the first job in each buffer is served by the weighted service power.

Since packets can not be divided in practice, a packetby-packet transmission scheme called packetized generalized processor sharing (PGPS) is presented to approximate GPS in [2]. The basic principle of PGPS is as follows: when the server is ready to transmit, and assuming no packet arrivals after the current time, it picks the packet which would complete the service in the GPS scheduler first among all packets. In an integrated service network, best effort services are often assigned with a low priority. The capacity for these low priority services are time varying as they can only use the remaining capacity from high priority services.

Let $r(k)$ be the varying system capacity at time instant $k, \Delta k$ be the time interval, $\mathbf{B}(K)$ be the set of backlogged sessions at time instant $k$, and $S_{i}(k, k+\Delta k)=\int_{k}^{k+\Delta k} r_{i}(s) d s$ be the amount of service received by the $i t h$ session in the time interval $[k, k+\Delta k]$. The GPS scheme allocates the service rate to the ith session according to the following rule:

$$
r_{i}(k)=\left\{\begin{array}{cc}
r(t) \phi_{i} / \sum_{j \in B(k)} \phi_{j} & \text { if } i \in B(k) \\
0 & \text { otherwise }
\end{array} .\right.
$$

Moreover, for any session $i$, that is continuously backlogged in the interval $[k, k+\Delta k]$, the received service should be

$$
S_{i}(k, k+\Delta k) \geq \frac{\phi_{i}}{\sum_{j=1}^{N} \phi_{j}} \int_{k}^{k+\Delta k} r(s) d s
$$

To implement GPS in an OFDM system, in each frame, we regard the total number of bits in the frame as the server power $P$. There are $N$ sessions and the $i$ th session is assigned a positive weight $\phi_{i}$. A session is said to be backlogged at time instant $k$ if there are packets of that session queued in the buffer at time instant $k$. We assume time interval $\Delta k$ is an integral multiple of the frame time $T_{\text {Frame }}$.

Since the packet length is variable, the number of packets accommodated in a frame is not fixed. In this case, the actual capacity $r(k)$ of the GPS server is variable depending on the actual number of bits in the current frame. The time-varying capacity $r(k)$ is piecewise constant between two successive frames. According to [2], under the new clock, the time $a_{i}^{l}$ is transformed to $w\left(a_{i}^{l}\right)$, and the virtual time $V(w(k))$ in the original PGPS should be modified as follows:

$$
\begin{gathered}
w(k)=0, \text { whenever } k \text { is not in a busy period } \\
w\left(k_{j-1}+\Delta k\right)=w\left(k_{j-1}\right)+r\left(k_{j-1}\right) \Delta k
\end{gathered}
$$

$$
V(w(k))=0, \text { whenever } k \text { is not in a busy period }
$$

$$
\begin{gathered}
V\left(w\left(k_{j-1}\right)=V\left(w\left(k_{j-1}\right)\right)+r\left(k_{j-1}\right) \Delta k / \Sigma_{j \in B(k)} \phi_{j}\right. \\
\Delta k \leq k_{j}-k_{j-1}, j=2,3 \ldots
\end{gathered}
$$

Let $a_{i}^{l}$ be the $l$ th packet arriving from the $i$ th user and $L_{i}^{l}$ be its packet size. When it arrives, it is stamped with the virtual finishing time

$$
\begin{gathered}
F_{i}^{l}=\max \left[F_{i}^{l-1}, V\left(w\left(a_{i}^{l}\right)\right)\right]+\frac{L_{i}^{l}}{\phi_{i}} \\
\text { with } F_{i}^{0}=0 \text { for all } i .
\end{gathered}
$$

\section{B. CPLD-PGPS Algorithm Description}

In this sub-section, we describe the algorithm of the CPLDPGPS scheduler. In order to guarantee fairness, we set a fairness value $V$ for each user in the system. All $V$ values are set to 0 at start time.

Step 1: In this scheduler, we only consider head-of-line packet for each user. When a packet arrives, by equation $(1,2,3)$, the virtual time is updated and the packet is stamped with its virtual finishing time.

Step 2: Based on the virtual finishing time of the headof-line packet in each queue, the scheduler serves packets in an increasing order of virtual finishing time. The packets are arranged in the frame until the remaining subcarriers are not enough to accommodate the next packet. If the number of remaining subcarriers $N_{r}$ is not small, we can make use of these vacant subcarriers. For each packet in the queue, the length is known. In the order of increasing finishing times, the scheduler will choose a packet which can be accommodated in the remaining subcarriers. This operation will increase the system throughput. In order to be fair to all users, subtract 1 from the fairness value $V$ of this user.

Step 3: Now the scheduler has finished packet selection. Due to the time varying channel of each user, it is necessary to optimize subcarrier allocation to different packets in the frame. We assume $N$ sub-carriers can be used for data transmission. So the total number of subcarriers is fixed. The subcarrier allocation function represents the subcarrier allocation

$$
f(p, n)= \begin{cases}1, & \text { if subcarrier } n \text { is allocated } \\ & \text { to the packet } p \\ 0, & \text { otherwise }\end{cases}
$$


Due to fading on different subcarriers, the power received on each subcarrier is different. Let $P_{T}$ be the total transmit power which is a constant and adaptively allocated to each subcarrier, $P_{p, n}$ be the transmit power allocated to packet $p$ on the nth subcarrier, and $P_{R, n}$ be the received power on the $n$th sub-carrier. The relationship between $P_{p, n}$ and $P_{R, n}$ can be expressed as

$$
P_{R, n}=P_{p, n}\left|H_{p, n}\right|^{2} .
$$

The BER performance on each subcarrier is related to the received power by

$$
\begin{aligned}
B E R_{n} & =\Gamma\left(P_{R, n}\right) \\
& =\Gamma\left(P_{p, n}\left|H_{p, n}\right|^{2}\right)
\end{aligned}
$$

As $P_{R, n}$ increases, BER decreases. As BER decreases, PER decreases. Thus the more received power in all subcarriers, the less system PER.

In this algorithm, our objective is to minimize BER, and hence minimize PER. This solution can optimally allocate the subcarriers to all packets in the frame. Let $\xi(k)$ be the set of packets in the frame at time $k, \psi$ be the total number of packets in the frame, $N_{b}$ be the number of bits in a subcarrier of the frame, $L_{p}$ be the packet length which is an integral multiple of $N_{b}$, and $N_{\text {packet }}$ be the total number of sub-carriers occupied by this packet. The optimal resource management problem in the OFDM frame can be described as :

$$
\left\{\begin{array}{l}
\text { Min } \sum_{p=1}^{\psi} \sum_{n=1}^{N} \Gamma\left(P_{p, n}\left|H_{p, n}\right|^{2}\right) f(p, n) \\
\text { Subject to } \\
\sum_{p} \sum_{n} f(p, n) \leq N(I) \\
\sum_{p=1}^{\psi} \sum_{n=1}^{N} P_{p, n}=P_{T}(I I) \\
f\left(p_{1}, n\right) f\left(p_{2}, n\right)=0 ; \forall p_{1} \neq p_{2} \in \xi(k),(I I I) \\
\sum_{n=1}^{N} f(p, n)=\left\lceil L_{p} / N_{b}\right\rceil ; \forall p(I V)
\end{array}\right.
$$

where $H_{p, n}$ denotes the channel gain of the $n$th subchannel of packet $p$. Condition $I$ states that the total sub-carriers allocated to all the users are less than or equal to $N$. Condition $I I$ shows that the total transmit power is fixed as $P_{T}$. Condition $I I I$ means that each subcarrier can only be allocated to one packet in a frame. Condition $I V$ says that the number of subcarriers allocated to the packet is related to the packet's length.

The solution of (8) will give the optimal subcarrier allocation function $f(p, n)$ in the frame. Since the channel information $H_{p, n}$ is provided and $P_{T}$ is adaptively allocated on each subcarrier to minimize the total BER performance, then $\Gamma\left(P_{p, n}\left|H_{p, n}\right|^{2}\right)$ is independent of $f(p, n)$. We get the solution in two steps.

First, according to the optimal subcarrier allocation algorithm in [14], an initial allocation is obtained via a constructive algorithm. We sort the subcarriers' gains for each user in a descending order, and we consider the first subcarriers in the ordered lists of all users, user by user. Suppose we consider the $n$th subcarrier of user $k$. If user $k$ 's subcarrier requirement in condition $I V$ has not been satisfied, and subcarrier $n$ has not been allocated to another user, then subcarrier $n$ is allocated to user $k$. Otherwise, skip to the next user. This operation continues until all the sub-carriers are allocated. So each packet gets the number of subcarriers it needs. Let
$\left\{D_{1}, D_{2} \ldots D_{\psi}\right\}$ be the set of subcarriers each packet occupies and $D_{\text {left }}$ be the set of idle subcarriers in the frame. Now function $f(p, n)$ is known to the scheduler. As described in [14], the next operation is iterative improvement. In each iteration, we try to swap the subcarriers allocated between two users with the objective of minimizing the transmission power. A cost function for swapping two users is calculated, which is used to select the pairs of users $A, B$ and the corresponding subcarriers in sets $D_{A}, D_{B}$. With the cost function, we get all power reduction factors. We sort all power reduction factors in descending order and pick the first one in the list. The iteration stops when all power reduction factors are negative. The swapping algorithm in the $\mathrm{C}$ language is given in Fig. 2. According to the optimal function $f(p, n)$, the scheduler allocates subcarriers to the packets.

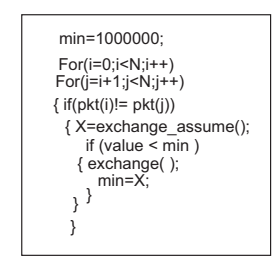

Fig. 2. Sub-carriers allocation optimization in the frame.

Second, the scheduler allocates the total transmission power onto each subcarrier. Since the objective of the scheduler is to minimize the BER performance, based on the initial subcarrier allocation scheme, the power is optimally allocated as follows. Based on the known function $f(p, n)$, equation (8) is simplified as

$$
\left\{\begin{array}{l}
\text { Min } \sum_{p=1}^{\psi} \sum_{n=1}^{N} \Gamma\left(P_{p, n}\left|H_{p, n}\right|^{2}\right) f(p, n) \\
\text { Subject to } \\
\sum_{p=1}^{\psi} \sum_{n=1}^{N} P_{p, n}=P_{T}
\end{array} .\right.
$$

With standard optimization techniques [20], we obtain the Lagrangian

$$
\begin{aligned}
L= & \sum_{p=1}^{\psi} \sum_{n=1}^{N} \Gamma\left(P_{p, n}\left|H_{p, n}\right|^{2}\right) f(p, n) \\
& -\lambda\left(P_{p, n}\right)\left(\sum_{p=1}^{\psi} \sum_{n=1}^{N} P_{p, n}-P_{T}\right)
\end{aligned}
$$

where $\lambda\left(P_{p, n}\right)$ is the Lagrangian multiplier for the constraint $I$. After differentiating $L$ with respect to $P_{p, n}$, with the total transmission power constraint we can get the optimal power allocation scheme.

Step 4: We check the goodness of each subcarrier allocated to each packet [24], count the total number of good subchannels $N_{\text {good }}$, and calculate the ratio $r$ between $N_{\text {good }}$ and $N_{\text {packet }}$. If the $r$ value of each packet in the frame is larger than $r_{0}$, which is pre-determined in system design, the frame will be sent out. For a packet whose $r$ value is smaller or equal to $r_{0}$, if the fair value $V$ is equal to or larger than $V_{0}$, which means it has given too many chances to others, the packet should be sent out anyway. Otherwise, packet exchange is needed. For example, assume $r \leq r_{0}$ and $V \geq V_{0}$ for packet $A$ in the frame, packet $B$ in the frame has the smallest $V$ value, and packet $C$ in the queues has the largest $V$ value, then packet $B$ should be exchanged with packet $C$; the $V$ value of packet 


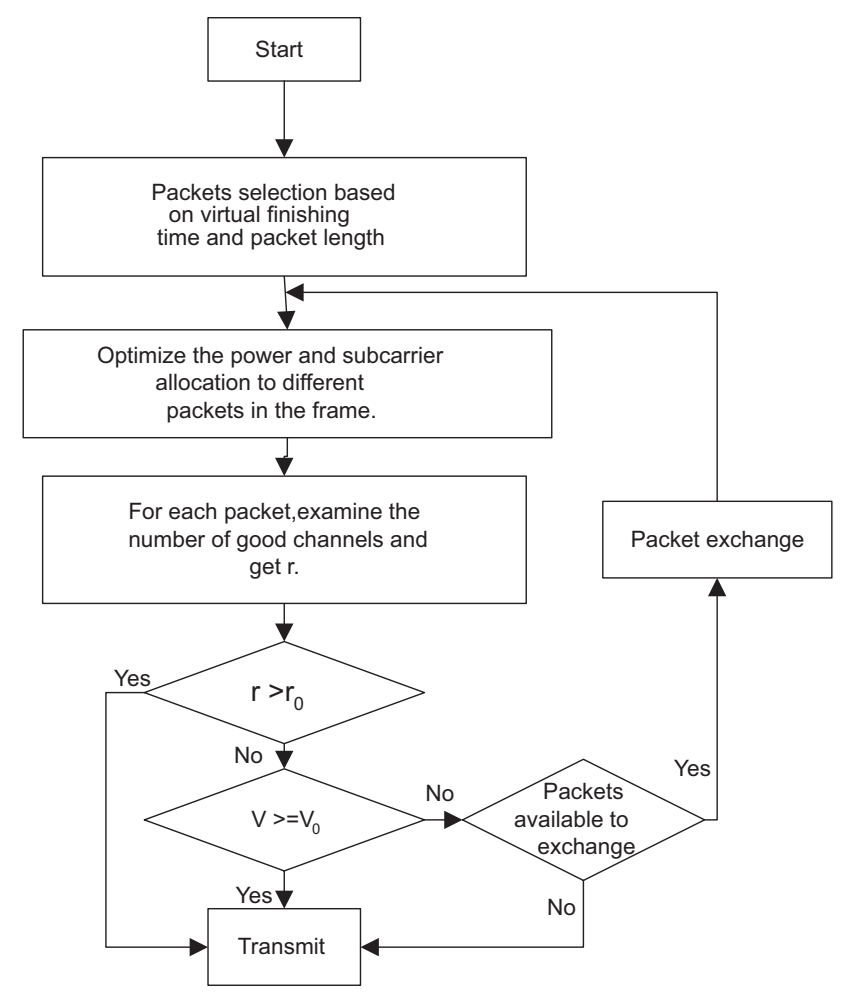

Fig. 3. The program flow.

$B$ 's user is incremented by 1 , while 1 is subtracted from the $V$ value of packet $C$ 's user; then return to Step 3. If the packet with the largest $V$ value (in the example packet $C$ ) can not fit in the subcarriers vacated by packet $B$, the algorithm will select the packet in the frame with the next largest $V$ value, and so on.

In this scheduler, both the channel condition and the packet length affect the scheduling result. If the packet length is short, and the number of good subchannels needed is also small, even though the total channel quality is not good, it is easy to find a set of good subchannels for the packet. From the algorithm, we find that the performance of the scheduler actually depends on the parameter $r_{0}$. The flow chart is presented in Fig. 3.

In practice, if the packet transmitted experiences errors and is detected by the receiver, this packet will be retransmitted, and this retransmission will waste system resource. This CPLD scheduler can guarantee the quality of packet transmission and reduce resource waste.

\section{Discussions}

If the server transmits the packet in the stamped finishing time order [2], it is fair for every user. However, if the channel condition does not allow packet transmission, the packet is deferred, and the transmission opportunity is given to other packets. Thus, it is unfair for that user. But with the fair value $V$, the scheduler will keep long term fairness. When a packet is deferred, the $V$ value is incremented by 1 , and the scheduler will select another packet whose fair value is the largest. Once a packet is transmitted, the fair value also needs to be decremented by 1 . Since we assume the wireless physical channels around the base station are statistically identical for all users, CPLD is fair in the long term.
There is another condition we should consider. If the wireless channel of the user remains unsatisfactory for a long time period, no packet is transmitted for this user. Then we set a threshold value $V_{0}$, if the fair value $V$ is larger than or equal to $V_{0}$, the packet must be included in the current frame anyway. This operation will avoid the "starving" phenomena, but the transmission quality of these packets can not be guaranteed. In practice, the wireless channel is divided into a great number of subchannels in the frequency domain, it is not very difficult for the packet to find enough good subchannels.

Since the packet length considered in this paper is variable, in each OFDM frame there may be a little number of subcarriers left empty. In order to deal with this problem, a packet can be divided into two small packets, one of which can be accommodated in the frame. So in this way, all subcarriers can be effectively utilized, and CPLD can be made work conserving. But this procedure needs to segment the packet, which increases the complexity. Actually it is a trade off between system throughput and complexity.

Compared with wireline networks, wireless channels always experience high transmission errors. Once the errors happen, and the packet can not be transmitted successfully, the network upper layer will require packet retransmission. So the wireless resource is wasted. The CPLD scheduler proposed in this paper optimizes the packet error rate (PER) performance according to the channel condition and packet length. So the probability of packet retransmission ratio is much reduced, and the whole system throughput is improved.

\section{SimPLified CPLD-PGPS}

The CPLD-PGPS algorithm described above will give optimal resource management and improve the system performance. However, the computational complexity is very high. In the algorithm, the packets are selected to fill in the OFDM frame, and the subchannels are optimally allocated to different packets, then packets may be exchanged, until the frame is filled and then sent out.

\section{A. Simplified CPLD-PGPS Algorithm}

To reduce the implementation complexity, we propose a simplified CPLD-PGPS scheduler.

Step 1: When the packet arrives at the queue, the scheduler will calculate the finishing time, and stamp it on the packet.

Step 2: The scheduler will select the packet of each session to fill the OFDM frame. Different from the above, the selection is based not only on the finishing time, but also on the parameter $r_{0}$. Since the packet length is known, and the modulation of each subcarrier is fixed, we can calculate the number of subcarriers $N_{\text {packet }}$ that should be allocated to this packet. We can also get the total number of good subchannels $N_{\text {good }}^{\prime}$ for this user. In the OFDM system, if one subchannel is a good channel for one user, it may also be a good channel for other users. So there is competition for good channels between users. For a packet, the good subchannels may not be all allocated to it after subcarrier allocation optimization. When we design the system, this point should be considered. We denote $N_{\text {good }}^{\prime} / N_{\text {packet }}$ by $r$. If $r$, is larger than $r_{0}^{\prime}$, the packet should be sent in the frame. Here $r_{0}^{\prime}$ is also set by the 


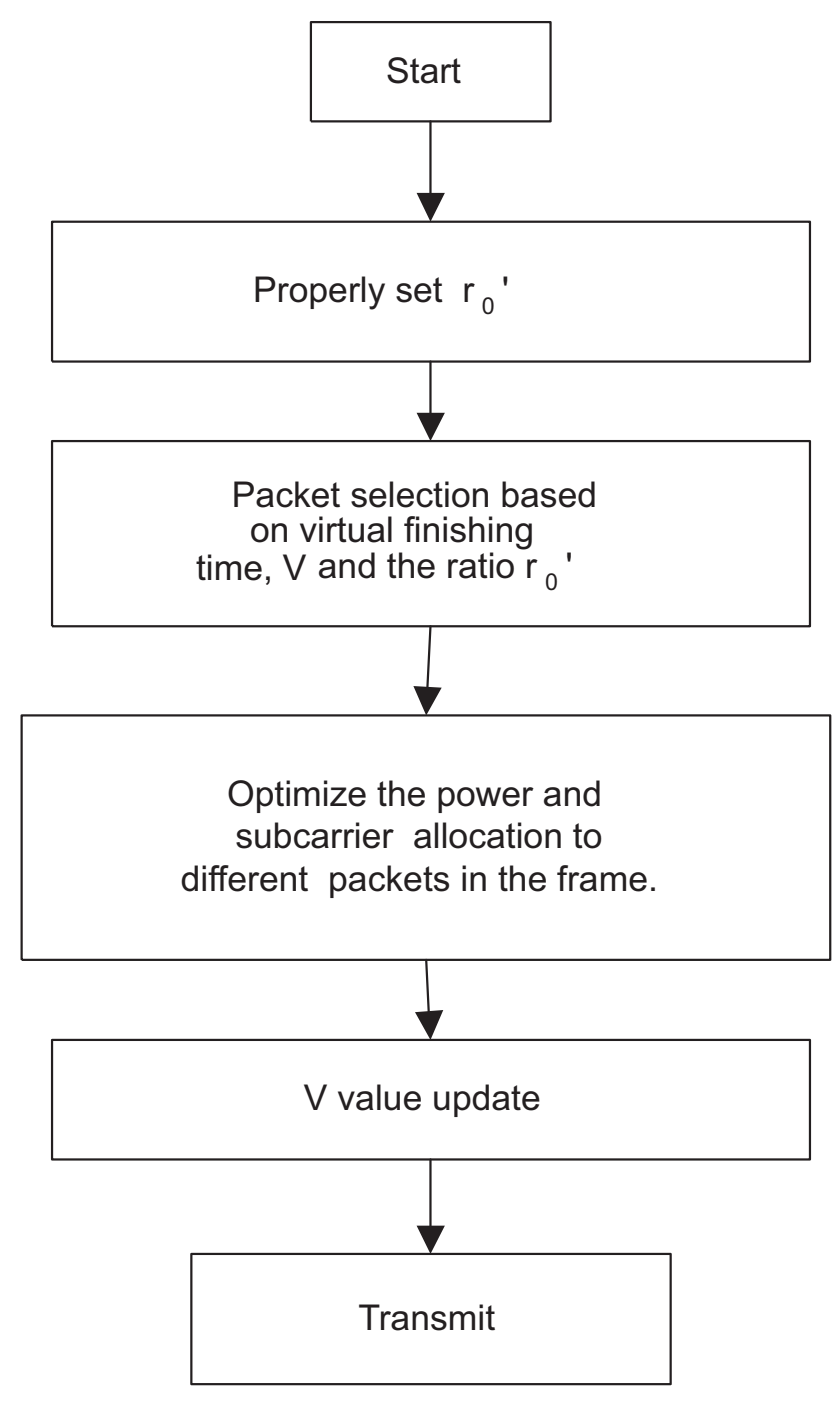

Fig. 4. The simplified program flow.

system and should be a little larger than $r_{0}$. If the fair value $V$ is larger than or equal to $V_{0}$, the packet should be selected in this frame.

According to the finishing time and the $r$, value, the packets are selected to fill the OFDM frame until the remaining subcarriers can not accommodate the next packet. In the order of increasing finishing times, the scheduler will choose a packet which can be accommodated in the remaining subcarriers, and its $r$, is also satisfied. All $V$ values of users whose head-of-line packet's finishing times are smaller than this packet should be incremented by 1 . This operation will improve the system throughput.

Step 3: After optimal subcarrier allocation in the frame, the transmission power is adaptively allocated on each subcarrier as in (8). Then the frame is transmitted.

In this simplified algorithm, the computational complexity is reduced. Further, system performance can be guaranteed by properly setting the system parameters $r_{0}$. The flow charter of the simplified CPLD scheduler is shown in Fig. 4.
TABLE I

COMPLEXITY EXPRESSION

\begin{tabular}{|c|c|}
\hline Complexity & Number of operations \\
\hline CPLD & $\mathrm{N}_{\mathrm{ex}}\left(\mathrm{N}_{\mathrm{U}} !+\mathrm{N}_{\mathrm{L}}\left(\mathrm{N}-\mathrm{N}_{\mathrm{L}}\right)+1+\mathrm{N}\right)+\mathrm{N}_{\mathrm{P}} \mathrm{C}_{\mathrm{PGPS}}$ \\
\hline $\begin{array}{c}\text { Simplified } \\
\text { CPLD }\end{array}$ & $\mathrm{N}_{\mathrm{U}} !+\mathrm{N}_{\mathrm{L}}\left(\mathrm{N}-\mathrm{N}_{\mathrm{L}}\right)+1+\mathrm{N}+\mathrm{N}_{\mathrm{P}} \mathrm{C}_{\mathrm{PGPS}}$ \\
\hline PGPS & $\mathrm{N}_{\mathrm{P}} \mathrm{C}_{\mathrm{PGPS}}$ \\
\hline
\end{tabular}

\section{B. Complexity Comparison}

Complexity of a scheduler is important for practical systems. In this subsection, the complexity comparison between the simplified CPLD-PGPS and CPLD-PGPS scheduler is given. As a measure of complexity, we count the number of operations each algorithm needs to fill the packet in an OFDM frame. Since the scheduler is based on the PGPS scheduler, we only count the operations beyond what PGPS needs. Let $N_{F}$ be the number of packets accommodated in a frame, $N_{e x}$ be the number of packets exchanged, and $N_{u}$ the number of users with packets. Since the scheduler is based on PGPS, the time stamp is updated. Let $C_{P G P S}$ be the complexity of virtual finishing time calculation of one packet in the queue. Then the complexity to get the virtual finishing time is $N_{P} C_{P G P S}$, where $N_{P}$ is the number of packets in the queue. For CPLD, according to the virtual finishing times, the packets are selected, then the subcarriers are optimally allocated. The number of subcarriers a packet occupies can be calculated as $N_{L}=\left\lceil L_{p} / N_{b}\right\rceil$. Then the number of operations for subcarriers swapping is $N_{L}\left(N-N_{L}\right)$. The good subchannels are counted, the parameter $r$ is calculated, and the $V$ value is updated. If the channel condition is not satisfied, the packet is exchanged. For simplified CPLD scheduler, the packet selection is not only based on virtual finishing time, but also the parameter $r^{\prime}$. The complexity comparison is shown in Table I and Fig. 5. $C_{P G P S}$ is set to 3 , and the average packet exchange time is set to 3 . There are 10 users having packets, and each only has two packets. With Fig. 5, it is found that CPLD-PGPS is much more complex than PGPS, but the complexity is much reduced by the simplified algorithm.

\section{Simulation Results}

In this section, we introduce the simulation conditions and the traffic generation model. Then the simulation results are given. The new scheduler performance is compared with PGPS.

\section{A. Simulation Setup}

In this paper, we consider an OFDM downlink system using QPSK modulation. The total system bandwidth is $20 \mathrm{MHz}$, which is divided into 256 sub-carriers, among which 192 are used for data. Each OFDM symbol lasts $4 \mu \mathrm{s}$, in which $0.8 \mu \mathrm{s}$ is the guard interval. We group 250 OFDM symbols into a frame, so the frame length is $1 \mathrm{~ms}$. Based on QPSK modulation, each 


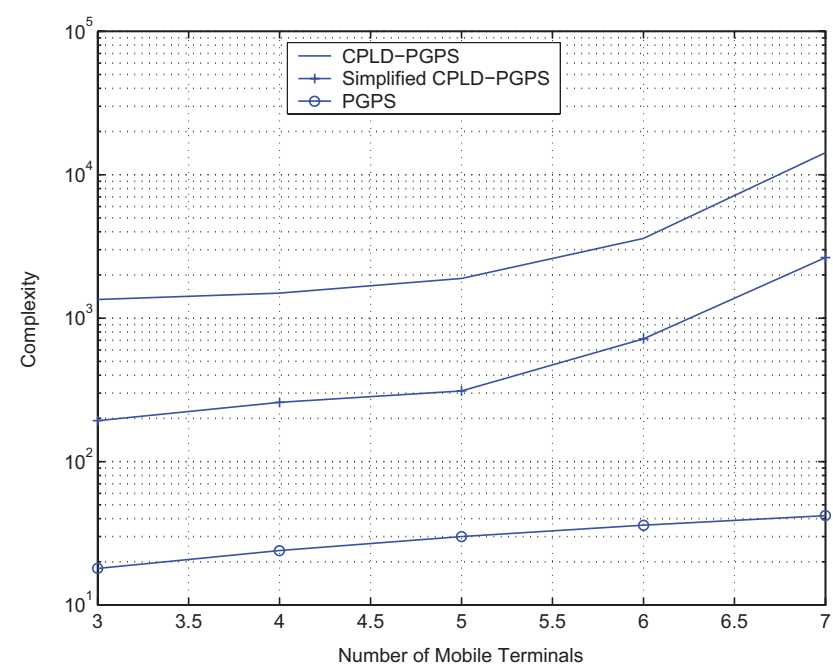

Fig. 5. Computational complexity of different schemes.

TABLE II

Voice Traffic Model Parameter

\begin{tabular}{|c|c|}
\hline State & $\begin{array}{c}\text { Average Duration } \\
\text { Time (s) }\end{array}$ \\
\hline $\begin{array}{c}\text { Principal } \\
\text { Talkspurt }\end{array}$ & 1.00 \\
\hline $\begin{array}{c}\text { Principal } \\
\text { Gap }\end{array}$ & 1.35 \\
\hline Minispurt & 0.275 \\
\hline Minigap & 0.05 \\
\hline
\end{tabular}

subcarrier in a frame can accommodate $N_{b}=2 \times 10^{3}$ bits. The packet length is set to be an integral multiple of $N_{b}$, and should be between $1.5 \times 10^{4}$ and $3 \times 10^{4}$ bits. We consider the quasistatic flat fading channel with multipath [23]. The multipath channel for each antenna has 6 taps of Rayleigh-faded paths at an interval $0.05 \mu \mathrm{s}$, and the power delay profile follows a decay rule of $\left[1, e^{-1}, e^{-2}, e^{-3}, e^{-4}, e^{-5}\right]$. Different users have independent channels with the same statistics. Forward error control coding is also applied in this system. The simulation time is 2 minutes.

\section{B. Traffic Model}

In the simulation, video, voice and data traffics are considered, and the corresponding traffic models are introduced next.

Video traffic is modeled by an 8-state Markov-Modulated Poisson Process (MMPP) [21]. In each state, the packet arrival satisfies a Poisson process. The average dwell time in each state is $40 \mathrm{~ms}$. The bit generation rate for different states is exponentially distributed.

The voice traffic generation model is the On-Off model. The speech source creates a pattern of talk spurts and gaps. In our simulation, the model is based on the three-state Markov

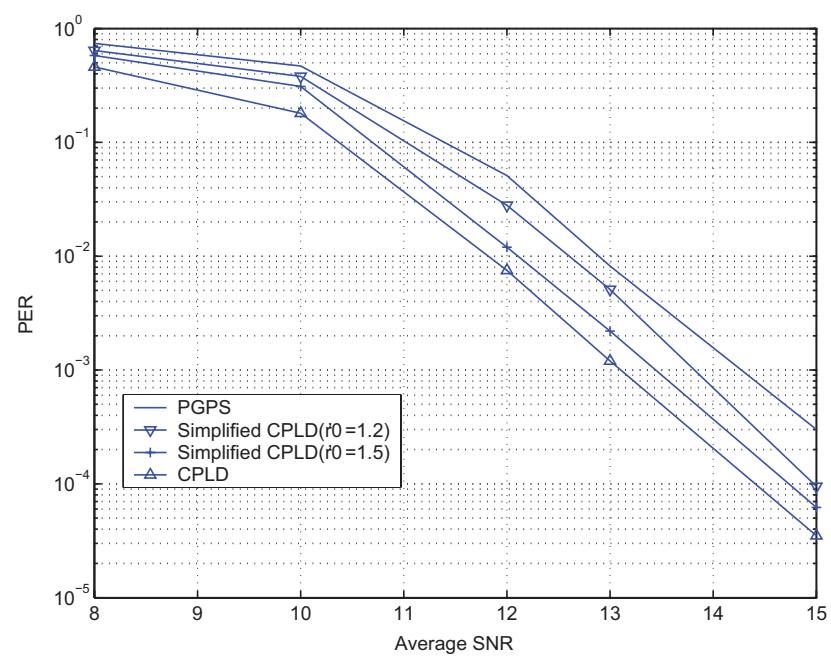

Fig. 6. PER performance comparison.

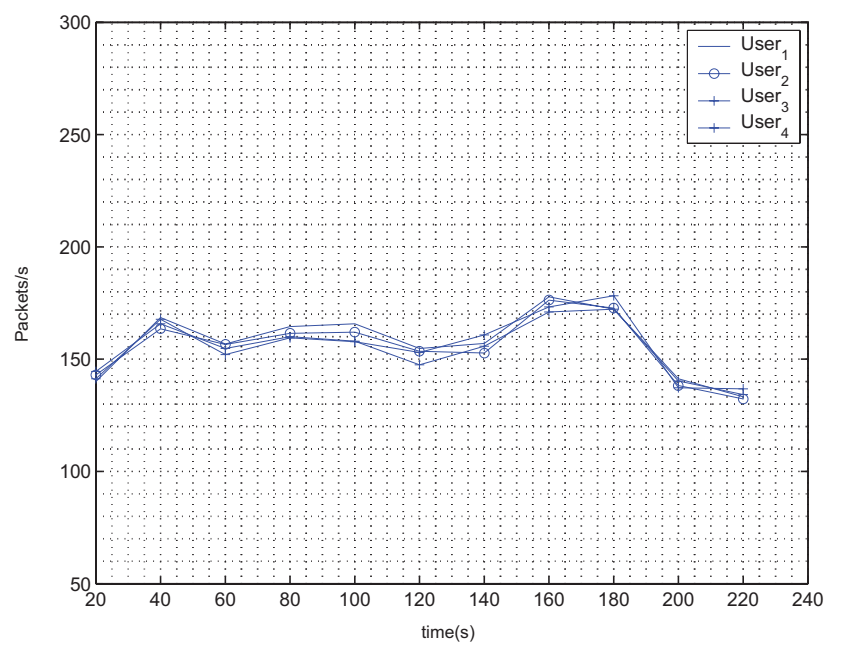

Fig. 7. Fairness comparison.

model presented in [18]. The duration of all spurts and gaps are exponentially distributed, and independent of each other. During the spurt states, we assume the mobile model generates a data rate of $16 \mathrm{~kb} / \mathrm{s}$. All the parameters of this model are listed in Table II. The data packet arrival also satisfies a Poisson process, and the mean inter-arrival time is $0.1 \mathrm{~s}$.

\section{Simulation Results}

First, four homogeneous video traffics are simulated with the same weight, $\phi_{1}=\phi_{2}=\phi_{3}=\phi_{4}$. The PGPS scheduler [7] with varying capacity is also simulated for performance comparison. For CPLD, the system parameter $r_{0}$ is set to 1. The wireless channel is normalized, and the parameter $E_{0}$ is set to 0.3. In wireless transmission, AWGN noise is added. The PER performance is given in Fig. 6. Since CPLD considers the wireless channel quality and the packet length at the same time, the performance is better than PGPS. The PER performance of simplified CPLD scheduler is greatly affected by the system parameter $r_{0}^{\prime}$. From the figure, we find that the system performance is improved as $r_{0}^{\prime}$ increases. By comparing CPLD and the simplified CPLD, we find that the 


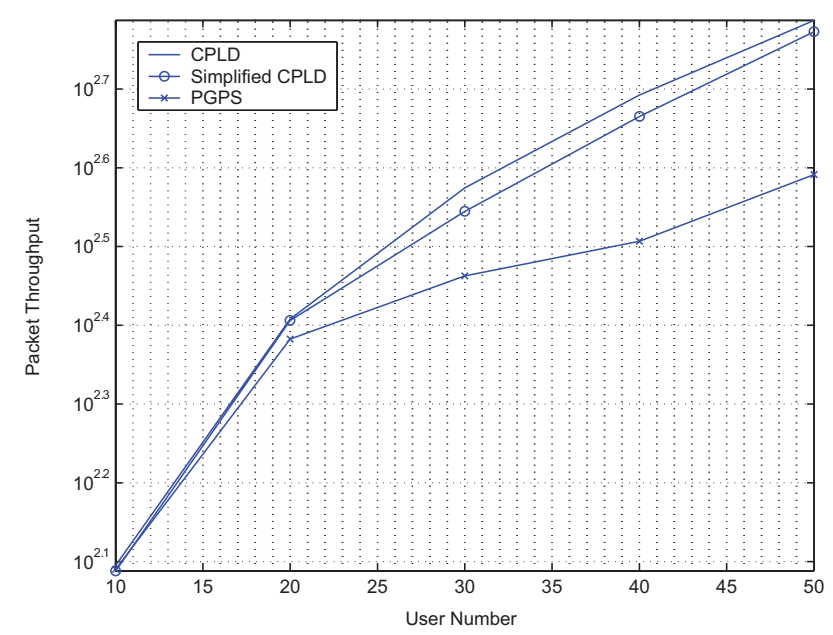

Fig. 8. Throughput comparison.

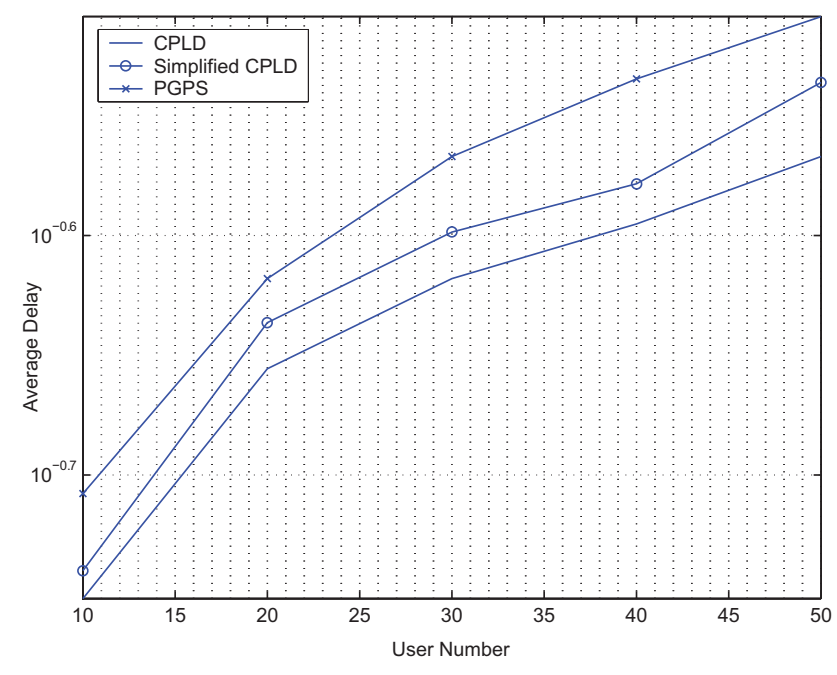

Fig. 9. Average delay comparison.

complexity of the scheduler is reduced while the performance can be guaranteed by properly designed parameters.

The fairness of the proposed CPLD scheduler is demonstrated in Fig. 7 in terms of the packets transmitted over a time duration of $20 \mathrm{~s}$. As the four users have the same weight, the average transmission rates are nearly the same, except for small fluctuations due to the bad channel conditions and the chance compensations. With the simulation results, it is found that the proposed CPLD scheduler can achieve nearly the same fairness performance as ideal PGPS.

To demonstrate the performance of the proposed resource management scheme in a heterogeneous traffic environment, we simulate a system in which voice, video, data traffic flows are transmitted at the same time. The voice traffic occupies $40 \%$ of the total traffic, while video and data traffic each occupies $30 \%$. The weight of the voice flows, video flows and data flows are set to be 3,2 , and 1, respectively. The parameter $r_{0}^{\prime}$ in the simplified CPLD scheduler is set to 1.5. The SNR of the system is set to $12 \mathrm{~dB}$.

We define the system packet throughput as the average number of packets successfully transmitted per minute. Fig. 8 gives the throughput of CPLD, simplified CPLD and PGPS.

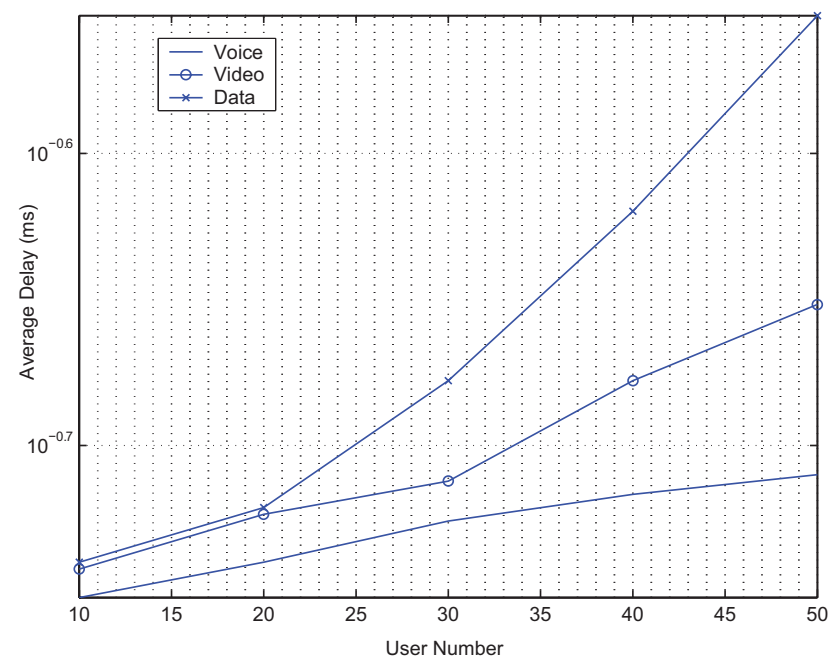

Fig. 10. Multimedia delay comparison.

It is found that the throughput of CPLD is much higher than PGPS, and the simplified CPLD throughput performance can be guaranteed by properly setting the parameter $r_{0}^{\prime}$.

Then the system average delay is compared in Fig. 9. When we calculate the average delay, if the packet is not successfully transmitted, the packet should be retransmitted, and the additional delay should also be considered. It is found that CPLD has better average delay performance than PGPS.

In the simulation, there are three types of traffic flow, and different traffics have different weights. A larger weight means higher priority. The average packet delays of different traffics are shown in Fig. 10. It can be seen that lower average transmission delays are achieved for voice traffic flow, since it has larger weight than video and data traffic flows.

\section{CONCLUSION AND FUTURE WORK}

In this paper, a new packet scheduler for OFDM systems is proposed. Based on PGPS, this scheduler considers the channel condition and the packet length simultaneously in OFDM systems. With fixed total transmission power, the sub-carriers are optimally allocated to different packets in the frame, and the power is also optimally allocated. Compared with PGPS, CPLD has better PER performance and system throughput. In order to reduce the complexity, a simplified CPLD is given, and PER performance can be achieved by properly setting the system parameters, while maintaining system throughput. Complexity is also compared among CPLD, simplified CPLD and PGPS. Simulation results have demonstrated that the proposed scheduler is suitable for supporting heterogeneous traffic flows and can achieve better system performance than PGPS in terms of PER, throughput, and delay. In addition, long term fairness is also provided for each user.

In this paper, we focus on scheduling in centralized networks. Extending these algorithms to distributed networks is a great challenge. All mobile users need to have the topology and channel information about the next several hops, and only based on such information can the centralized scheduling algorithms be extended to distributed networks. More research is needed in this area. 


\section{ACKNOWLEDGMENT}

This research is supported in part by the Research Grants Council of the Hong Kong Special Administrative Region, China (Project No. HKU 7152/05).

\section{REFERENCES}

[1] A. K. Parekh and R. G. Gallager, "A generalized processor sharing approach to flow control in integrated services networks: The singlenode case," IEEE/ACM Trans. Networking, vol. 1, no. 3, pp. 344-357, June 1993

[2] Y. C. Tseng and C. S. Chang, "PGPS servers with time-varying capacities," IEEE Commun. Lett., vol. 2, no. 9, pp. 266-268, Sep. 1998.

[3] S. Lu and V. Bharghava, "Fair scheduling in wireless packet networks," IEEE/ACM Trans. Networking, vol. 7, pp. 473-489, Aug. 1999.

[4] Y. Cao, V. O. K. Li, and Z. Cao, "Scheduling delay-sensitive and besteffort traffics in wireless networks," in Proc. IEEE ICC, May 2003, vol. 3, pp. 2208-2212.

[5] P. Bhagwat, P. Bhattacharya, A. Krishna, and S. K. Tripathi, "Enhancing throughput over wireless LANS using channel state dependent packet scheduling," in Proc. IEEE INFOCOM, Mar. 1996, vol. 3, pp 11331140.

[6] Y. Cao and V. O. K. Li, "Scheduling algorithms in broad-band wireless networks," Proc. IEEE, vol. 89, no. 1, pp. 76-87, Jan. 2001.

[7] Y. Cao and V. O. K. Li, "Adaptive scheduling and coding for timevarying links," in Proc. ICCT, Apr. 2003, vol. 2, pp. 1384-1387.

[8] W. Chen, K. Shih, and J. Chiang, "Flexible packet scheduling for quality of service provisioning in wireless networks," in Proc. 9th International Conf. Parallel Distributed Syst., Dec. 2002, pp. 381-387.

[9] J. Capone and L. Stavrakakis, "Delivering QoS requirements to traffic with diverse delay tolerances in a TDMA environment," IEEE/ACM Trans. Networking, vol. 7, no. 1, pp. 75-87, Feb. 1999.

[10] L. Zhuge, Y. Cao, and V. O. K. Li, " Adaptive packet scheduling in cellular CDMA,” in Proc. IEEE PIMRC 2003, Sep. 2003, vol. 2, pp $1192-1196$.

[11] L. Xu, X. Shen, and J. W. Mark, " Dynamic fair scheduling with QoS constraints in multimedia wideband CDMA cellular networks," IEEE Trans. Wireless Commun., vol. 3, no. 1, pp. 60-73, Jan. 2004.

[12] M. A. Arad and A. L. Garcia, "A generalized processor sharing approach to time scheduling in hybrid CDMA/TDMA," in Proc. IEEE INFOCOM, Mar. 1998, pp. 1164-1171.

[13] S. B. Weinstein and P. M. Ebert, "Data transmission by frequencydivision multiplexing using the discrete fourier transform," IEEE Trans. Commun., vol. 19, pp. 628-634, Oct. 1971.

[14] Y. Y. W. Cheong, C. Y. Tsui, R. S. Cheng, and K. B. Letaief, "A realtime sub-carrier allocation scheme for multiple access downlink OFDM transmission," in Proc. IEEE VTC, Sep. 1999, vol. 2, pp. 1124-1128.

[15] Y. W. Cheong, R. S. Cheng, K. B. Letaief, and R. D. Murch, "Multiuser OFDM with adaptive subcarrier, bit, and power allocation," IEEE $J$. Select. Areas Commun., vol. 17, no. 10, pp. 1747-1758, Oct. 1999.

[16] L. Xiao, A. Wang, S. Zhou, and Y. Yao, "A dynamic resource scheduling algorithm for OFDM system," in Proc. APCC, Sep. 2003, vol. 2, pp. 444-447.

[17] W. Anchun, X. Liang, Z. Shidong, X. Xinbin, and Y. Yan, "Dynamic resource management in the fourth generation wireless systems," in Proc. ICCT, Apr. 2003, vol. 2, pp. 1095-1098.

[18] J. Cai, S. Shen, and J. W. Mark, "Downlink resource management for packet transmission in OFDM wireless communication systems," in Proc. IEEE Globecom, Dec. 2003, vol. 6, pp. 2999-3003.

[19] J. Chuang and N. Sollenberger, "Beyond 3G: Wideband wireless data access based on OFDM and dynamic packet assignment," IEEE Commun. Mag, vol. 38, no. 7, July 2000.

[20] D. G. Luenberger, Optimization by Vector Space Methods. New York: Wiley, 1969.

[21] D. J. Goodman and S. X. Wei, "Efficiency of packet reservation multiple access," IEEE Trans. Veh. Technol., vol. 40, pp. 170-176, Feb. 1991.

[22] H. Seo and B. G. Lee, "A proportional-fair power allocation scheme for fair and efficient multiuser OFDM systems," in Proc. IEEE Globecom, Nov. 2004, vol. 6, pp. 3737-3741.

[23] C. Xiao, Y. Zheng, and N. Beaulieu, "Second-order statistical properties of the WSS Jakes' fading channel simulator," IEEE Trans. Commun., vol. 50, no. 6, pp. 888-891, June 2002.

[24] A. J. Goldsmith and S.-G. Chua, "Variable-rate variable-power MQAM for fading channels," IEEE Trans. Commun., vol. 45, no. 10, pp. 12181230, Oct. 1997

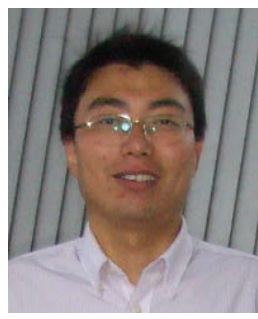

Zhifeng Diao (S'04) received the BS and MS from Xidian University, Xi'an, China, in 1999 and 2002 respectively, and $\mathrm{PhD}$ from the University of Hong Kong, Hong Kong, China in 2005. Currently, he is a postdoctoral fellow at the Department of Electrical Engineering, the University of California, Riverside, CA, USA. His research interests include wireless scheduling algorithm design, OFDM, MC-CDMA, cross layer design, smart antennas.

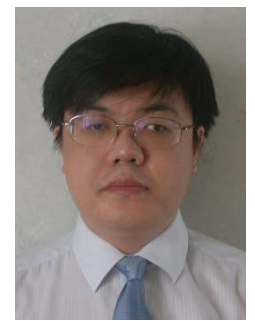

optimization.

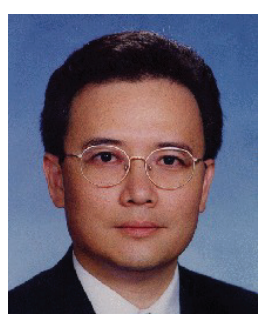

Dongxu Shen (S'97-M'01) received the BS from Harbin University of Engineering, Harbin, China, in 1995, MS and PhD from Rensselear Polytechnic Institute, Troy, New York, USA, in 1999 and 2001 respectively, all in Electrical Engineering. From Dec. 2001 to April 2005, he was a Research Associate and from May 2005, he is a PostDoc Fellow, both at the Department of Electrical and Electronic Engineering, The University of Hong Kong, Hong Kong. His research interests include smart antenna, OFDM systems, media access, scheduling, and cross-layer

Victor O. K. Li (S'80-M'81-SM'86-F'92) (http://www.eee.hku.hk/staff_personal/vli.htm) received SB, SM, EE and ScD degrees in Electrical Engineering and Computer Science from the Massachusetts Institute of Technology, Cambridge, Massachusetts, in 1977, 1979, 1980, and 1981 respectively. He joined the University of Southern California (USC), Los Angeles, California, USA in February 1981, and became Professor of Electrical Engineering and Director of the USC Communication Sciences Institute. Since September 1997 he has been with the University of Hong Kong, Hong Kong, where he is Chair Professor of Information Engineering at the Department of Electrical and Electronic Engineering. He has also served as Managing Director of Versitech Ltd. (http://www.versitech.com.hk/), the technology transfer and commercial arm of the University, and on various corporate boards. His research is in information technology, including all-optical networks, wireless networks, and Internet technologies and applications. He is a Principal Investigator of the Area of Excellence in Information Technology funded by the Hong Kong government.

Sought by government, industry, and academic organizations, he has lectured and consulted extensively around the world. Prof. Li chaired the Computer Communications Technical Committee of the IEEE Communications Society 1987-1989, and the Los Angeles Chapter of the IEEE Information Theory Group 1983-1985. He co-founded the International Conference on Computer Communications and Networks (IC3N), and chaired its Steering Committee 1992-1997. He also chaired various international workshops and conferences, including, most recently, IEEE INFOCOM 2004. Prof. Li has served as an editor of IEEE Network, IEEE JSAC Wireless Communications Series, and Telecommunication Systems. He also guest edited special issues of IEEE JSAC, Computer Networks and ISDN Systems, and KICS/IEEE Journal of Communications and Networking. He is now serving as an editor of ACM/Kluwer Wireless Networks and IEEE Communications Surveys and Tutorials.

Prof. Li has been appointed to the central policy unit and to the Hong Kong Information Infrastructure Advisory Committee by the Chief Executive of the Hong Kong Special Administrative Region. He also serves on the Innovation and Technology Fund (Electronics) Vetting Committee, the Small Entrepreneur Research Assistance Programme Committee, the Engineering Panel of the Research Grants Council, and the Task Force for the Hong Kong Academic and Research Network (HARNET) Development Fund of the University Grants Committee. He was a Distinguished Lecturer at the University of California at San Diego, at the National Science Council of Taiwan, and at the California Polytechnic Institute. Prof. Li has also delivered keynote speeches at many international conferences. $\mathrm{He}$ has received numerous awards, including, most recently, the Ministry of Education Chang Jiang Chair Professorship at Tsinghua University, Beijing, the UK Royal Academy of Engineering Senior Visiting Fellowship in Communications, the Outstanding Researcher Award of the University of Hong Kong, the Croucher Foundation Senior Research Fellowship, and the Bronze Bauhinia Star, Government of the Hong Kong Special Administrative Region, China. He was elected an IEEE Fellow in 1992. He is also a Fellow of the HKIE and the IAE. 